\title{
Radiation Therapy against Pediatric Malignant Central Nervous System Tumors : Embryonal Tumors and Proton Beam Therapy
}

\author{
Do Hoon Lim, M.D., Ph.D. \\ Department of Radiation Oncology, Samsung Medical Center, Sungkyunkwan University School of Medicine, Seoul, Korea
}

Radiation therapy is highly effective for the management of pediatric malignant central nervous system (CNS) tumors including embryonal tumors. With the increment of long-term survivors from malignant CNS tumors, the radiation-related toxicities have become a major concern and we need to improve the treatment strategies to reduce the late complications without compromising the treatment outcomes. One of such strategies is to reduce the radiation dose to craniospinal axis or radiation volume and to avoid or defer radiation therapy until after the age of three. Another strategy is using particle beam therapy such as proton beams instead of photon beams. Proton beams have distinct physiologic advantages over photon beams and greater precision in radiation delivery to the tumor while preserving the surrounding healthy tissues. In this review, I provide the treatment principles of pediatric CNS embryonal tumors and the strategic improvements of radiation therapy to reduce treatment-related late toxicities, and finally introduce the increasing availability of proton beam therapy for pediatric CNS embryonal tumors compared with photon beam therapy.

Key Words : Child · Neoplasm · Brain · Radiation therapy · Proton beam therapy.

\section{INTRODUCTION}

In recent decades, radiation therapy has changed greatly through the development of radiation delivery and treatment planning systems. Like other cancer treatment modalities, the main purposes of radiation therapy are to maximize the local tumor control and minimize the treatment-related late toxicities. Especially for children, radiation therapy should be carefully applied because the radiation tolerance of children is much lower than that of adult patients and the late effect of radiation will last forever.
Among the pediatric solid tumors, central nervous system (CNS) embryonal tumors are medulloblastomas, supratentorial primitive neuroectodermal tumors (CNS embryonal tumour, not otherwise specified [NOS] in 2016 World Health Organization [WHO] classification) and atypical teratoid/ rhabdoid tumors (AT/RTs). The basic principle of treatment for CNS embryonal tumors is maximal tumor resection followed by radiation therapy and chemotherapy. However, CNS embryonal tumors can be most strongly affected by radiation because the incidence is highest in young children aged 1-4 years and radiation therapy targeting whole neuroaxis is one

- Received : December 29, 2017 •Revised : February 4, 2018 •Accepted : February 14, 2018

- Address for reprints : Do Hoon Lim, M.D., Ph.D.

Department of Radiation Oncology, Samsung Medical Center, 81 Irwon-ro, Gangnam-gu, Seoul 06351, Korea

Tel : +82-2-3410-2600, Fax : +82-2-3410-2609, E-mail : dh8lim@skku.edu

This is an Open Access article distributed under the terms of the Creative Commons Attribution Non-Commercial License (http://creativecommons.org/licenses/by-nc/4.0) which permits unrestricted non-commercial use, distribution, and reproduction in any medium, provided the original work is properly cited. 
of the standards of treatments.

To reduce the treatment-related late toxicities without compromising the outcomes, treatment strategies have developed directing to intensification of chemotherapy and decrease of radiation dose or irradiated volume. In addition, the introduction of particle beams such as proton beam therapy for pediatric tumors has increased during the last decade.

In this review, the principles of managements for pediatric CNS embryonal tumors and the strategic developments to reduce treatment-related late toxicities are provided, and the proton beam therapy for CNS embryonal tumors is introduced and compared with photon beam therapy.

\section{CNS EMBRYONAL TUMORS AND TREATMENT}

CNS tumors are the most common types of solid tumors in children and it accounts for $20-25 \%$ of all pediatric malignancies. CNS embryonal tumors constitute about $20 \%$ of all pediatric CNS tumors and medulloblastoma is the most common type of CNS embryonal tumors. Therefore, medulloblastoma is the most common pediatric malignant CNS tumor and it represents $15-20 \%$ of pediatric CNS tumors and $40 \%$ of posterior fossa tumors ${ }^{8)}$.

Medulloblastoma patients are stratified into standard-risk or high-risk group according to the age at presentation, the presence of anaplasia, the extent of postoperative residual tumor and the presence of tumor dissemination into the cerebrospinal fluid $(\mathrm{CSF})^{20)}$. Standard-risk of medulloblastoma is defined by postoperative minimal residual tumor $\left(<1.5 \mathrm{~cm}^{2}\right)$, an age of 3 years or older, no anaplasia, and no evidence of tumor dissemination into the CSF. Likewise, high-risk medulloblastoma is defined by postoperative residual tumor more than $1.5 \mathrm{~cm}^{2}$ or an age of less than 3 years old or presence of anaplasia, or tumor dissemination into the CSF.

Supratentorial primitive neuroectodermal tumors (CNS embryonal tumour, NOS in 2016 WHO classification) have a similar biology to high-risk medulloblastoma and are considered as high-risk embryonal tumors. AT/RTs typically present in very young children less than 2 years old ${ }^{28)}$ and are diagnosed on the basis of a characteristic immunohistochemical lack of nuclear INI1 protein expression. They are associated with a high risk of early relapse and a very poor prognosis and also considered as high-risk embryonal tumors.
The treatment of pediatric malignant CNS tumors is still challenging because it requires a multidisciplinary approach and the treatment principles are maximal tumor resection followed by radiation therapy and chemotherapy. However, the radiation dose or volume and chemotherapeutic agents or dose intensification are modified according to the risk of tumor.

Standard-risk medulloblastoma patients were initially treated with 36 gray (Gy) of craniospinal irradiation (CSI) and posterior fossa (PF) boost up to 54 Gy with a daily dose of $1.8 \mathrm{~Gy}$ over 6 weeks in postoperative setting. However, the CSI dose reduction studies revealed that CSI dose would be reduced 23.4 Gy from 36 Gy without compromising treatment outcomes ${ }^{18,27)}$.

The early studies for adjuvant chemotherapy have failed to show the additional effect on postoperative radiation therapy alone, but the current recommendations of chemotherapy for patients with standard-risk medulloblastoma are concurrent radiochemotherapy using weekly vincristine and approximately one year of maintenance chemotherapy consisting of cisplatin, lomustine (cyclonexyl-chloroethyl-nitrosourea), and vincristine. There have been several studies with neoadjuvant chemotherapy prior to irradiation, but the delays in the initiation of radiation therapy have been associated with inferior outcomes.

Radiation therapy for high-risk medulloblastoma in children 3 years of age and older is 36 Gy of CSI with a boost to both PF and metastatic sites to 55.8 Gy. For chemotherapy, the ideal chemotherapeutic regimens have not been identified yet. However, the treatment results for high-risk medulloblatoma are disappointing and 5 -year survival is less than $55 \%{ }^{7,25,30)}$. Therefore, to overcome the unsatisfactory treatment results, the intensification of chemotherapy using high-dose chemotherapy and autologous stem cell transplantation (HDCT/autoSCT) has been tried and shown some clinical benefit in children with highrisk or recurrent solid tumors ${ }^{9,11)}$. With this scheme of managements using HDCT/autoSCT and reduced-dose of CSI (23.4 Gy or 30.6 Gy instead 36 Gy), Korean researchers showed 70\% of 5-year event-free survival (EFS) in high-risk medullblastoma patients $^{23)}$.

For high-risk CNS embryonal tumors developing in children younger than 3 years old, the neurocognitive outcomes are very poor after radiation therapy including CSI. Therefore, avoiding or deferring radiation therapy until after the age of 3 has been issued and the intensified chemotherapy strategies, which were the addition of systemic or intraventricular methotrexate to 
postoperative induction chemotherapy or the use of HDCT/ autoSCT, have been investigated. Those studies showed a satisfactory outcome in some populations of children but resulted in early local recurrence. In recurrent cases, salvage radiation therapy showed tumor response, and some studies applying the early local radiation therapy instead CSI even in children less than 3 years old have been investigated to avoid the toxicities of CSI as well as reduce early local recurrences ${ }^{21,26)}$.

\section{RADIATION THERAPY FOR CNS EMBRYONAL TUMORS}

At the time of diagnosis, about $10-40 \%$ of medulloblastoma patients develop leptomeningeal seeding and it can metastasize extracranially such as bone. Because of high risk of dissemination to CSF, CSI is the major radiation therapy strategy for CNS embryonal tumors. After CSI, the boost irradiation to PF (or primary tumor bed) or metastatic sites should be followed. Historically, the standard radiation dose is 54-55.8 Gy including 36 Gy for craniospinal axis and 18-19.8 Gy boost for PF or primary tumor bed. However, during the last decades, the results of many clinical studies showed that it was possible to reduce CSI dose or radiation volume and to avoid or defer radiation therapy until after the age of 3 while maintaining or improving survival rates and decreasing radiation-induced late toxicities.

\section{CSI dose reduction}

In a phase III randomized trial by Pediatric Oncology Group and Children's Oncology Group $(\mathrm{COG})^{27)}$, a reduced-dose of CSI (23.4 Gy) was compared with a standard-dose of CSI (36 Gy) and the conclusion was that reduced-dose neuraxis irradiation was associated with increased risk of early neuraxis relapse, and lower 5-year EFS and overall survival (OS) than the standard-dose of irradiation. However, after the subsequent study ${ }^{18)}$ which demonstrated that the addition of chemotherapy to reduced-dose of CSI prolonged survival, the reduced-dose of CSI (23.4 Gy) strategy is now considered as the standard radiation for standard-risk medulloblastoma patients. For high-risk medulloblastoma, there was a study trying to decrease CSI dose with the intensification of chemotherapy ${ }^{1)}$, but 36 Gy of CSI dose remains as the standard for high-risk medulloblastoma.

According to COG study that investigated the intellectual outcomes after the reduction of CSI dose from 36 Gy to 23.4
Gy, the decline in intelligence quotient was still substantial and the amounts of decline were 4.3 points per year. However, some degree of intellectual preservation was observed when compared with those associated with conventional radiation doses $^{22)}$. Furthermore, a subsequent CSI dose-reduction study showed that a reduced CSI dose of 18.0-23.4 Gy resulted in stable cognitive outcomes at 5 years after treatment ${ }^{14)}$. Therefore, further CSI dose reduction trials continue to be investigated.

COG ACNS 0331 was a randomized study to determine whether reducing the CSI dose from 23.4 Gy to 18 Gy in young children aged 3 to 7 years dose not compromise EFS and OS (Fig. 1). The final results are not published, however, reduced dose of craniospinal axis irradiation was associated with higher event rates and worse survival. Therefore, 23.4 Gy of CSI is the standard radiation dose for standard-risk medulloblastoma.

\section{Reduction of radiation volume}

Despite neuroaxis dose reduction, the cognitive function decline continues to be a significant treatment-related toxicity and

Standard risk (3-7 years)

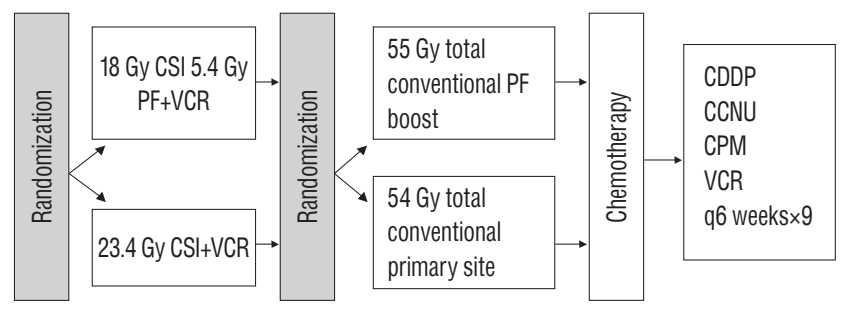

\section{(A)}

Standard risk ( $\geq 8$ years)

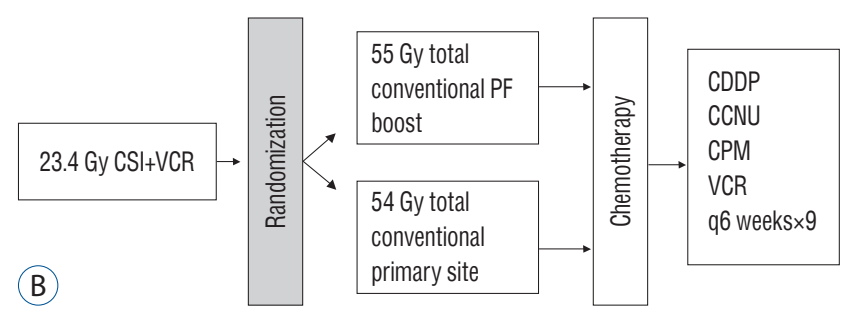

Fig. 1. COG ACNS 0331 study diagram for standard-risk medulloblastoma. For children aged 3 to 7 years, CSI dose (23.5 Gy vs. 18.0 Gy) and boost radiation volume (primary site only versus entire posterior fossa) are randomized (A). For children aged 8 years or more, only boost radiation volume is randomized (B). COG : Children's Oncology Group, CSI : craniospinal irradiation, PF : posterior fossa, VCR : vincristine, CDDP : cisplatin, CCNU : lomustine, CPM : cyclophosphamide. 
new radiation targeting guidelines to limit the boost volume to the primary site only was suggested. Several studies have demonstrated that the PF boost volume may be safely reduced to the primary tumor bed, allowing for preservation of normal organs such as the temporal lobes, hypothalamus, and cochleae ${ }^{13,29)}$.

COG ACNS 0331 study was a randomized study to determine if reducing the irradiated volume of the primary site tumor boost from the whole posterior fossa to the tumor bed only will not compromise EFS and OS regardless of age (Fig. 1). Survival rates following reduced radiation boost volumes were comparable to standard treatment volumes for the primary tumor site and radiation oncologist can adopt smaller boost volume for posterior fossa irradiation.

\section{Avoidance or deferral of radiation therapy for young children less than 3 years old}

CNS embryonal tumors in young children are associated with worse survival than in older children, because it is not easy to avoid disease progression with a limited use of radiation related with functional impairment of developing brain in younger ages. Therefore, treatment strategy using HDCT/autoSCT without radiation therapy or with deferring radiation therapy have been tried and showed positive results in highrisk or recurrent brain tumors. The recent studies suggested that further dose intensification of HDCT/autoSCT might improve the outcomes.

Some clinical trials using intensified chemotherapy in infants and young children with malignant CNS tumors made it possible to avoid or defer radiation therapy without negatively affecting survival rates ${ }^{2,5,10,24)}$. However, in case of AT/RT, disease progression developed early in the course of treatment and radiation therapy might be more efficacious than chemotherapy, even for very young children ${ }^{21,26)}$. Therefore, up-front radiation therapy is typically used early after surgery and can vary from localized therapy to CSI, depending on the patient's age and the extent of disease.

\section{PROTON BEAM THERAPY VERSUS PHOTON BEAM THERAPY}

During radiation therapy, radiation exposure to normal brain surrounding the tumor is inevitable and it can lead to late toxicities and affect the quality of life for long-term survivors. The possible treatment-related sequelae after CNS irradiation include neurocognitive impairment, endocrine dysfunction, growth abnormality, and secondary malignancies ${ }^{4,6,15-17,19)}$. The Children Cancer Survivor Study revealed that survivors of childhood cancer have a high rate of illness due to chronic health conditions from treatment and the risk of long-term morbidities was threefold higher among adults treated with cranial irradiation for CNS tumors in childhood than in their siblings without cranial irradiation ${ }^{17)}$. Therefore, the development of radiotherapeutic modality or technique that can preserve more normal brain is one of the most considerable points for radiation oncologist. Especially for CNS embryonal tumors, it became more important because the entire craniospinal axis should be irradiated.

Photon ( $\mathrm{x}$-ray) beam radiation therapy is the conventional radiotherapeutic approach for pediatric tumors. High energy photon beams penetrate tissues and are targeted to the tumor to deliver therapeutic radiation dose. After the introduction of the advanced radiation delivery techniques such as three-dimensional conformal radiation therapy and intensity-modulated radiation therapy, the precision of radiation therapy has improved and radiation oncologist can deliver high dose of radiation to the tumor with minimizing the irradiated volume of normal tissues. However, the delivery of high dose to the tumor from multiple photon beams induces unwanted exposure of normal tissues to low to intermediate radiation dose, causing radiation-induced toxicities.

Otherwise, proton beam is basically distinct from photon beam. Charged particles such as proton make radiation oncologists handle the radiation more precisely and preserve normal tissues without compromising the radiation dose to the tumor. This is possible because of the physical property of proton beam named "Bragg peak" and the majority of proton's energy is released within a few millimeters of Bragg peak. Before the Bragg peak, proton loses a small amount of energy, so delivers a low 'entrance' dose. Beyond the Bragg peak, proton has no energy, so delivers no 'exit' dose (Fig. 2). The physical advantages of proton beam over photon beam can reduce radiation-induced toxicities and improve quality of life for patients who become long-term survivors of certain pediatric CNS tumors.

Fig. 3 shows the difference of delivered radiation dose from proton or photon beams when the whole spinal axis is irradiated for medulloblastoma patients. Proton beam has no exit 
dose beyond Bragg peak and there is little radiation to normal organs such as heart, liver, bowels which are located at the front of the radiation target.

Several studies reported the neurocognitive effect of proton beam therapy and compared with historical results of photon beam therapy. Generally those studies indicate that the use of

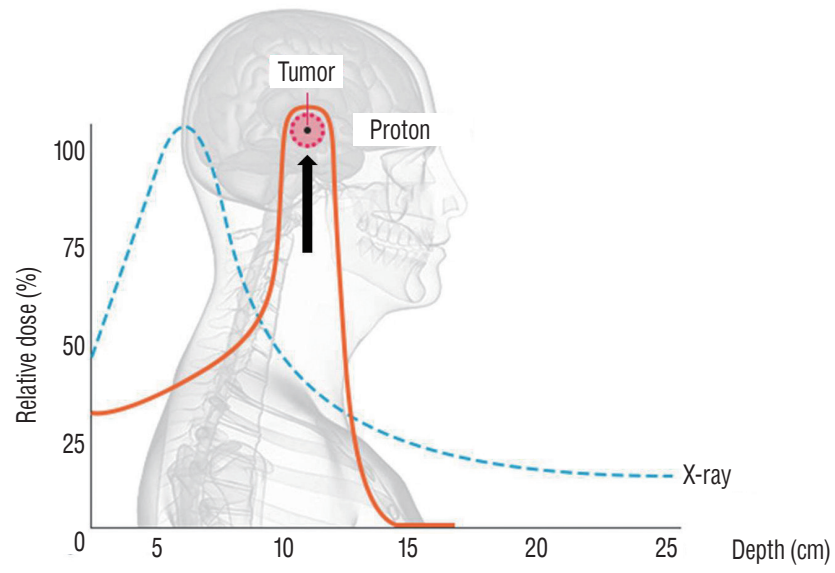

Fig. 2. Relative dose of proton and photon beams according to depth. The majority of proton's energy is released within a few millimeters of Bragg peak (black arrow). proton beams can mitigate some of the neurocognitive sequelae from radiation therapy for CNS tumors. In one study, proton beam therapy rather than photon beam therapy reduced the volume of supratentorial brain and temporal lobe that received low or intermediate doses of radiation, and differences in the overall dose distributions showed that a reduction in radiation dose or volumes would have long-term, clinical advantages $^{12)}$.

Another benefit of proton beam therapy over photon beam therapy is a reduction of risk for secondary malignancy and it might be the result of reduced radiation exposure of healthy tissue surrounding the tumor (Fig. 3). According to the Childhood Cancer Survivor Study ${ }^{1)}$, the cumulative incidence of all subsequent neoplasms in survivors of pediatric CNS tumors at 25 years after diagnosis is $10.7 \%$ although benign second neoplasms make up a significant proportion of these second tumors.

A matched cohort study ${ }^{3)}$ compared the risk of secondary malignancy in 558 patients treated with proton beam therapy at Harvard with that in another 558 patients' data treated with photon beam therapy from The Surveillance, Epidemiology, and End Results. Proton beam therapy was associated with a lower incidence of second malignancies than photon beam therapy (6.9



Fig. 3. Sagittal (A) and axial (B) computed tomography images showing radiation dose distributions. Red lines show the planning target volume and red color areas are regions irradiated with $100 \%$ of prescribed doses. Proton beam has no exit dose beyond Bragg peak and yellow arrows show that there is little radiation to normal organs such as heart, liver and bowels which are located at the front of the radiation target (red lines) in proton beam therapy. It can decrease the risk of second malignancies from radiation. 
vs. 10.3 per 1000 person-years, $p=0.085)$. In a dosimetric study for medulloblastoma ${ }^{31}$, researchers have calculated a risk of secondary malignancy after CSI with proton beam and photon beam. The predicted lifetime attributable risk was 4.6-10.0 fold higher with photon beam than with proton beam. These clinical and dosimetric studies strongly support the role of proton beam in diminishing the secondary malignancy risk, which is the most serious and life-threatening adverse effect of radiation for longterm survivors of pediatric malignant CNS tumors.

\section{CONCLUSION}

Survival in pediatric malignant CNS tumors continues to improve with the development of multidisciplinary approach and many efforts have been made to decrease treatment-related late toxicities. For radiation oncologists, these efforts included the developments of radiotherapeutic modality or techniques that can preserve more normal brain and the modification of radiation dose or irradiating volume according to tumor risk. In addition, the introduction of particle beams such as proton beam therapy for pediatric CNS tumors has a promise. The clinical application of proton beam therapy is increasing and the clinical results to date suggest that proton beam can reduce radiation-related late toxicities such as neurocognitive impairment and secondary malignancy. Therefore, proton beam therapy will become more widely available to children with malignant CNS tumors.

\section{CONFLICTS OF INTEREST}

No potential conflict of interest relevant to this article was reported.

\section{INFORMED CONSENT}

This type of study does not require informed consent.

\section{References}

1. Armstrong GT : Long-term survivors of childhood central nervous system malignancies: the experience of the Childhood Cancer Survivor Study. Eur J Paediatr Neurol 14 : 298-303, 2010

2. Chi SN, Gardner SL, Levy AS, Knopp EA, Miller DC, Wisoff JH, et al. : Feasibility and response to induction chemotherapy intensified with highdose methotrexate for young children with newly diagnosed high-risk disseminated medulloblastoma. J Clin Oncol 22 : 4881-4887, 2004

3. Chung CS, Yock TI, Nelson K, Xu Y, Keating NL, Tarbell NJ : Incidence of second malignancies among patients treated with proton versus photon radiation. Int J Radiat Oncol Biol Phys 87 : 46-52, 2013

4. Diller L, Chow EJ, Gurney JG, Hudson MM, Kadin-Lottick NS, Kawashima TI, et al. : Chronic disease in the Childhood Cancer Survivor Study cohort: a review of published findings. J Clin Oncol 27 : 2339-2355, 2009

5. Fangusaro J, Finlay J, Sposto R, Ji L, Saly M, Zacharoulis S, et al. : Intensive chemotherapy followed by consolidative myeloablative chemotherapy with autologous hematopoietic cell rescue (AuHCR) in young children with newly diagnosed supratentorial primitive neuroectodermal tumors (sPNETs): report of the Head Start I and II experience. Pediatr Blood Cancer $50: 312-318,2008$

6. Grill J, Renaux VK, Bulteau C, Viguier D, Levy-Piebois C, Sainte-Rose C, et al. : Long-term intellectual outcome in children with posterior fossa tumors according to radiation doses and volumes. Int J Radiat Oncol Biol Phys 45 : 137-145, 1999

7. Kortmann RD, Kühl J, Timmermann B, Mittler U, Urban C, Budach V, et al. : Postoperative neoadjuvant chemotherapy before radiotherapy as compared to immediate radiotherapy followed by maintenance chemotherapy in the treatment of medulloblastoma in childhood: results of the German prospective randomized trial HIT '91. Int J Radiat Oncol Biol Phys 46 : 269-279, 2000

8. Louis DN, Ohgaki H, Wiestler OD, Cavenee WK, Burger PC, Jouvet A, et al. : The 2007 WHO classification of tumours of the central nervous system. Acta Neuropathol 114 : 97-109, 2007

9. Marachelian A, Butturini A, Finlay J : Myeloablative chemotherapy with autologous hematopoietic progenitor cell rescue for childhood central nervous system tumors. Bone Marrow Transplant 41 : 167-172, 2008

10. Mason WP, Grovas A, Halpern S, Dunkel IJ, Garvin J, Heller G, et al. : Intensive chemotherapy and bone marrow rescue for young children with newly diagnosed malignant brain tumors. J Clin Oncol 16 : 210-221, 1998

11. Matthay KK, Reynolds CP, Seeger RC, Shimada H, Adkins ES, Haas-Kogan $D$, et al. : Long-term results for children with high-risk neuroblastoma treated on a randomized trial of myeloablative therapy followed by 13-cisretinoic acid: a children's oncology group study. J Clin Oncol 27 : 10071013, 2009

12. Merchant TE, Hua CH, Shukla H, Ying X, Nill S, Oelfke U : Proton versus photon radiotherapy for common pediatric brain tumors: comparison of models of dose characteristics and their relationship to cognitive function. Pediatr Blood Cancer 51 : 110-117, 2008

13. Merchant TE, Kun LE, Krasin MJ, Wallace D, Chintagumpala MM, Woo SY, et al. : Multi-institution prospective trial of reduced-dose craniospinal irradiation (23.4 Gy) followed by conformal posterior fossa (36 Gy) and primary site irradiation (55.8 Gy) and dose-intensive chemotherapy for average-risk medulloblastoma. Int J Radiat Oncol Biol Phys 70 : 
782-787, 2008

14. Moxon-Emre I, Bouffet E, Taylor MD, Laperriere N, Scantlebury N, Law N, et al. : Impact of craniospinal dose, boost volume, and neurologic complications on intellectual outcome in patients with medulloblastoma. J

Clin Oncol 32 : 1760-1768, 2014

15. Mulhern RK, Kepner JL, Thomas PR, Armstrong FD, Friedman HS, Kun LE : Neuropsychologic functioning of survivors of childhood medulloblastoma randomized to receive conventional or reduced-dose craniospinal irradiation: a Pediatric Oncology Group study. J Clin Oncol 16 : 1723-1728, 1998

16. Mulhern RK, Merchant TE, Gajjar A, Reddick WE, Kun LE : Late neurocognitive sequelae in survivors of brain tumours in childhood. Lancet Oncol 5 : 399-408, 2004

17. Oeffinger KC, Mertens AC, Sklar CA, Kawashima T, Hudson MM, Meadows AT, et al. : Chronic health conditions in adult survivors of childhood cancer. N Engl J Med 355 : 1572-1582, 2006

18. Packer RJ, Gajjar A, Vezina G, Rorke-Adams L, Burger PC, Robertson PL, et al. : Phase III study of craniospinal radiation therapy followed by adjuvant chemotherapy for newly diagnosed average-risk medulloblastoma. J Clin Oncol 24 : 4202-4208, 2006

19. Packer RJ, Gurney JG, Punyko JA, Donaldson SS, Inskip PD, Stovall M, et al. : Long-term neurologic and neurosensory sequelae in adult survivors of a childhood brain tumor: childhood cancer survivor study. J Clin Oncol 21 : 3255-3261, 2003

20. Packer RJ, Rood BR, MacDonald TJ : Medulloblastoma: present concepts of stratification into risk groups. Pediatr Neurosurg 39 : 60-67, 2003

21. Park ES, Sung KW, Baek HJ, Park KD, Park HJ, Won SC, et al. : Tandem high-dose chemotherapy and autologous stem cell transplantation in young children with atypical teratoid/rhabdoid tumor of the central nervous system. J Korean Med Sci 27 : 135-140, 2012

22. Ris MD, Packer R, Goldwein J, Jones-Wallace D, Boyett JM : Intellectual outcome after reduced dose radiation therapy plus adjuvant chemotherapy for medulloblastoma: a Children's Cancer Group study. J Clin Oncol 19 : 3470-3476, 2001

23. Sung KW, Lim DH, Son MH, Lee SH, Yoo KH, Koo HH, et al. : Reduceddose craniospinal radiotherapy followed by tandem high-dose chemo- therapy and autologous stem cell transplantation in patients with highrisk medulloblastoma. Neuro Oncol $15:$ 352-359, 2013

24. Sung KW, Lim DH, Yi ES, Choi YB, Lee JW, Yoo KH, et al. : Tandem highdose chemotherapy and autologous stem cell transplantation for atypical teratoid/rhabdoid Tumor. Cancer Res Treat 48 : 1408-1419, 2016

25. Taylor RE, Bailey CC, Robinson KJ, Weston CL, Walker DA, Ellison D, et al. : Outcome for patients with metastatic (M2-3) medulloblastoma treated with SIOP/UKCCSG PNET-3 chemotherapy. Eur J Cancer 41 : 727-734, 2005

26. Tekautz TM, Fuller CE, Blaney S, Fouladi M, Broniscer A, Merchant TE, et al. : Atypical teratoid/rhabdoid tumors (ATRT): improved survival in children 3 years of age and older with radiation therapy and high-dose alkylator-based chemotherapy. J Clin Oncol 23 : 1491-1499, 2005

27. Thomas PR, Deutsch M, Kepner JL, Boyett JM, Krischer J, Aronin P, et al. : Low-stage medulloblastoma: final analysis of trial comparing standarddose with reduced-dose neuraxis irradiation. J Clin Oncol 18 : 30043011,2000

28. Woehrer A, Slavc I, Waldhoer T, Heinzl H, Zielonke N, Czech T, et al. : Incidence of atypical teratoid/rhabdoid tumors in children: a populationbased study by the Austrian Brain Tumor Registry, 1996-2006. Cancer 116 : 5725-5732, 2010

29. Wolden SL, Dunkel IJ, Souweidane MM, Happersett L, Khakoo Y, Schupak $K$, et al. : Patterns of failure using a conformal radiation therapy tumor bed boost for medulloblastoma. J Clin Oncol 21 : 3079-3083, 2003

30. Zeltzer PM, Boyett JM, Finlay JL, Albright AL, Rorke LB, Milstein JM, et al. : Metastasis stage, adjuvant treatment, and residual tumor are prognostic factors for medulloblastoma in children: conclusions from the Children's Cancer Group 921 randomized phase III study. J Clin Oncol 17 : 832845, 1999

31. Zhang R, Howell RM, Taddei PJ, Giebeler A, Mahajan A, Newhauser WD : A comparative study on the risks of radiogenic second cancers and cardiac mortality in a set of pediatric medulloblastoma patients treated with photon or proton craniospinal irradiation. Radiother Oncol 113 : 8488,2014 